Research Article

\title{
The Refraction Correction of Elevation Angle for the Mean Annual Global Reference Atmosphere
}

\author{
Leke Lin (iD, Xiangming Chen, Ranran Hu, and Zhenwei Zhao \\ National Key Laboratory of Electromagnetic Environment, China Research Institute of Radiowave Propagation, \\ Qingdao 266107, China \\ Correspondence should be addressed to Leke Lin; 1lk22s@163.com
}

Received 19 January 2020; Accepted 23 April 2020; Published 7 May 2020

Academic Editor: Stefano Selleri

Copyright $(2020$ Leke Lin et al. This is an open access article distributed under the Creative Commons Attribution License, which permits unrestricted use, distribution, and reproduction in any medium, provided the original work is properly cited.

In some frequency-sharing studies between fixed service and space radiocommunication services, including fixed-satellite, broadcasting-satellite, and space science services, it is necessary to estimate the apparent elevation angle of a space station, taking into account the atmospheric refraction. Recommendations ITU-R (International Telecommunication Union-Radiocommunication) P.834-9 and F.1333-1 detail similar methods regarding calculating the refraction correction for the elevation angle of the mean annual global reference atmosphere. Herein, both methods are approximated using the bending angle from the ground to the infinity height; this approach is most suitable for geosynchronous orbit satellites. In this paper, new methods for calculating the refraction correction for the elevation angle are proposed regarding the mean annual global reference atmosphere given in Recommendation ITU-R P.835-6. Specifically, the results of the ray-tracing method are fitted. The height of the new formulae is $100 \mathrm{~km}$ above sea level. For higher altitudes, correction methods are given based on free-space propagation. The proposed methods can be applied to the calculation of the refraction correction for the elevation of the mean annual global reference atmosphere for satellites at different orbital heights. Furthermore, these new methods compare favourably to the two ITU-R Recommendations.

\section{Introduction}

A radio wave passing through the actual atmosphere travels along a curved line, rather than a straight one. The velocity of propagation of these waves will be lower than the velocity at which light travels in vacuum. All of these effects are referred together as the atmospheric refraction of a radio wave. Atmospheric refraction is a very significant topic regarding fields such as satellite communication, terrestrial microwave relay communication, fixed and mobile communication, and radio location services [1]. In some frequency sharing studies between the fixed service and space radiocommunication services, for example, including fixed-satellite, broadcastingsatellite, and space science services, it is necessary to estimate the apparent elevation angle of a space station. This involves taking the atmospheric refraction into account $[2,3]$.

The random variation of the atmospheric refractive index with respect to time and space, however, means that atmospheric refraction can exhibit extensive and complicated contents. The variation of the atmospheric refractive index with respect to height is usually far greater than its variation with respect to horizontal distance. Consequently, in general, it is postulated that the atmospheric refractive index is spherically stratified, while being horizontally uniform. In this case, tropospheric refractivity profiles become the fundamental data for correcting the tropospheric refractive error. Considering the planning and compatibility analysis of radio systems worldwide, it is necessary to have a reference standard atmosphere all over the world for use in the calculation of atmospheric refraction effects or gaseous attenuation, among other uses. Recommendation (Rec.) ITU-R P.835 provides expressions and data for reference standard atmospheres, which are based on the United States Standard Atmosphere (1976). The mean annual global reference atmosphere given in Rec. ITU-R P.835 was modified in 2017 (P.835-6, [4]) to explicitly define the pressure at the transitions between various layers. 
The refraction correction for the elevation angle can be calculated using an integral expression derived from ray theory $[1,5,6]$. However, for engineering applications, it is more convenient to establish a simplified calculation method to estimate the refraction correction for the elevation angle towards a space station, considering atmospheric refraction. Rec. ITU-R P.834-9 provides methods to calculate the refraction correction for the elevation angle of the mean annual global reference atmosphere in Section 4 (4.2 and 4.3). These methods include two scenarios: either the apparent elevation angle is known or the geometric elevation angle (i.e., the elevation angle under free-space propagation conditions) is known. There are also different methods with similar equations in Rec. ITU-R F.1333-1. Therefore, it is necessary to determine which method is preferable. Moreover, both of the methods given in the two Recommendations are approximated by the bending angle from the ground to the infinity height and are therefore most suitable for geosynchronous orbit satellites.

The methods detailed in Rec. ITU-R P.834-9 are in fact the same as those given in Rec. ITU-R P.834-2 [7], which were published in 1997. Meanwhile, the methods detailed in Rec. ITU-R P.834-9 are also the same as those detailed in Rec. ITU-R F.1333-0 [8], which were also published in 1997; these methods were subsequently modified in Rec. ITU-R F.1333-1 in 1999.

In this paper, new methods for calculating the refraction correction of elevation angle are proposed, using the mean annual global reference atmosphere given in Rec. ITU-R P.835-6, by fitting the results of the ray-tracing method. The height of the new formulae is determined to be $100 \mathrm{~km}$ above sea level. For higher altitudes, correction methods are detailed here based on free-space propagation. The proposed methods can be used to calculate the refraction correction for the elevation of the mean annual global reference atmosphere for satellites at different orbital heights. Furthermore, these new methods are shown here to outperform the methods given in the two ITU-R Recommendations.

This paper is organized as follows. The ray-tracing method to calculate the refraction correction for the elevation angle is detailed in Section 2. Section 3 introduces the new formulae for calculating the refraction correction for the elevation angle. Section 4 provides a comparison of the different methods. The conclusions are surmised in Section 5 .

\section{The Ray-Tracing Method for the Refraction Correction for the Elevation Angle of the New Mean Annual Global Reference Atmosphere}

It should be noted that the integral equation for the refraction correction, $\tau$, detailed in P.834-9 (5) and F.1333-1 (1), is not the refractive error of the elevation angle but is in fact the bending angle, which is shown in Figure 1. At heights of above several hundred kilometres to tens of thousands of kilometres (satellite), saturation of this bending will occur. The elevation angle error, $\varepsilon$, due to atmospheric refraction will then be nearly equal to the bending angle
( $\rho \approx 0 \Rightarrow \tau \approx \theta-\theta_{0}=\varepsilon$ ). For the sake of accuracy, this study uses the following analytical formulae to calculate the elevation angle error.

The ray-tracing technique used herein is briefly introduced here; it is referred in detail in Xie et al.'s study [1]. Assuming a spherically symmetric refractive medium, a ray is described by Snell's law as

$$
r n(r) \cos \theta=A \text {, }
$$

where $r=r_{e}+h$ is the distance from Earth's centre to the observation point, $A$ is a constant for a given ray, and $n$ is the refractive index (usually converted to refractivity $\left.N=(n-1) \times 10^{6}\right)$. Figure 1 shows a schematic presentation of atmospheric refraction.

$\phi$ can be given by [1]

$$
\phi=A \int_{r_{1}}^{r_{2}} \frac{d r}{r \sqrt{n^{2}(r) r^{2}-A^{2}}} .
$$

$\theta_{0}$ can be calculated according to the geometrical relation depicted in Figure 1 using the accurate position of point 2:

$$
\theta_{0}=\arctan \left(\frac{r_{2} \cos \phi-r_{1}}{r_{2} \sin \phi}\right) \text {. }
$$

Therefore, if the angles of $\theta$ and $\theta_{0}$ are known, then the refraction correction for the elevation angle can be obtained by

$$
\varepsilon=\theta-\theta_{0} .
$$

It should be noted that the denominator will be equal to 0 in (2) when the apparent elevation angle is $0^{\circ}$. In this case, an approximate approach is taken. (2) can be expressed as

$$
\begin{aligned}
\phi & =A \int_{r_{1}}^{r_{2}} \frac{d r}{r \sqrt{n^{2}(r) r^{2}-A^{2}}} \\
& =A \int_{r_{1}}^{r_{2}} \frac{d r}{r \cdot n(r) r \sqrt{1-\left(A^{2} / n^{2}(r) r^{2}\right)}} \\
& =A \int_{r_{1}}^{r_{2}} \frac{d r}{n(r) r^{2} \sqrt{1-\left(A^{2} / n^{2}(r) r^{2}\right)}} .
\end{aligned}
$$

Using the power series expansion formula,

$\frac{1}{\sqrt{1+x}}=1-\frac{1}{2} x+\frac{1 \cdot 3}{2 \cdot 4} x^{2}-\frac{1 \cdot 3 \cdot 5}{2 \cdot 4 \cdot 6} x^{3}+\cdots(-1<x \leq 1)$.

Then, the following can be obtained:

$$
\begin{aligned}
\phi & =A \int_{r_{1}}^{r_{2}} \frac{d r}{n(r) r^{2} \sqrt{1-\left(A^{2} / n^{2}(r) r^{2}\right)}} \\
& =A \int_{r_{1}}^{r_{2}} \frac{d r}{n(r) r^{2}}\left(1-\frac{1}{2} x+\frac{1 \cdot 3}{2 \cdot 4} x^{2}-\frac{1 \cdot 3 \cdot 5}{2 \cdot 4 \cdot 6} x^{3}+\cdots\right), \\
x & =\frac{A^{2}}{n^{2}(r) r^{2}} .
\end{aligned}
$$




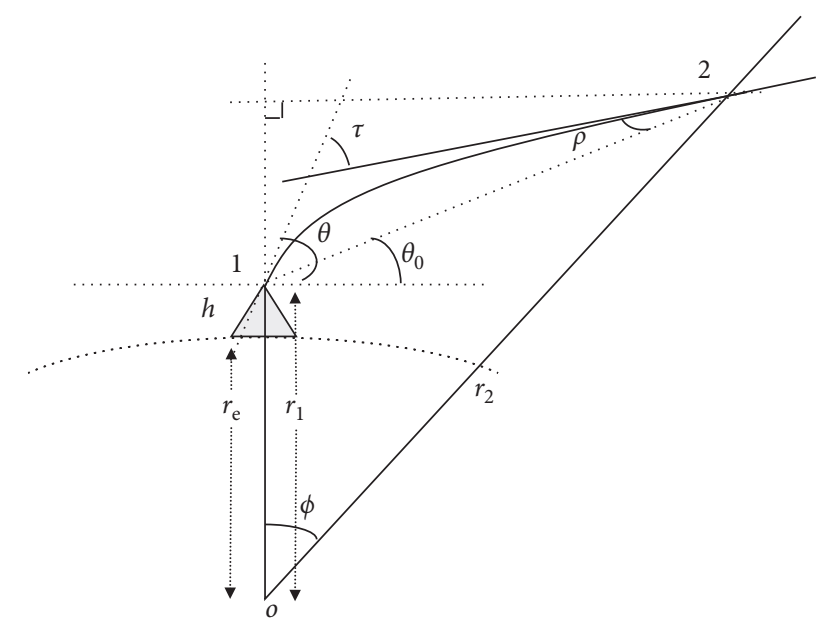

FIGURE 1: A schematic representation of atmospheric refraction. $\tau$ : bending angle, $\rho$ : angle between the tangent of point 2 and line segment 12, $\theta$ : apparent elevation angle, $\theta_{0}$ : geometric elevation angle, $r_{1}$ and $r_{2}$ : distances from Earth's centre to points 1 and 2, respectively, $\phi$ : spherical angle between points 1 and $2, r_{e}$ : radius of Earth, and $h$ : ray height above sea level.

A four-class series approximation (until $\left.x^{4}\right)$ is adopted in the actual calculation.

Neutral atmospheric refractivity profiles are the fundamental data for correcting the atmospheric refractive error regarding Earth-space links. The radio refractivity of the air in the troposphere can be obtained by ([9], note that the results of the two formulae ((6) and (7)) in the Recommendation are almost identical):

$$
N=77.6 \frac{P}{T}+3.732 \times 10^{5} \frac{e}{T^{2}},
$$

where $P$ is the atmospheric pressure $(\mathrm{hPa}), e$ is the water vapour pressure $(\mathrm{hPa})$, and $T$ is the absolute temperature (K).

It should be noted that

$$
P=P_{d}+e,
$$

where $P_{d}$ is the pressure of the dry atmosphere.

According to the above method, the refraction corrections of the elevation angle are compared with two kinds of methods for the mean annual global reference atmosphere, i.e., P.835-6 (new) and P.835-5 [10] (old). The atmospheric pressures provided by the Recommendations are considered to be the pressures of the dry atmosphere. The results are shown in Figure 2, in which the initial height of the calculation is set to $0 \mathrm{~km}$ above sea level. The abscissa indicates the apparent elevation angle, and the ordinate indicates the elevation angle error $(\varepsilon)$; both these parameters are expressed in degrees. In the calculation, the interval of the apparent elevation angle is $0.1^{\circ}$ below $10^{\circ}$, while the interval above $10^{\circ}$ is $1^{\circ}$. The maximum altitude is set to $100 \mathrm{~km}$, in which the refractivity is set to zero when the altitude is above $85 \mathrm{~km}$ for the old mean annual global reference atmosphere. Figure 2 shows that the difference between the two kinds of atmosphere is negligible, so the new mean annual global reference atmosphere was used in all subsequent calculations. It can also be seen from Figure 2 that the results are reasonable for the approximation displayed in (7).

When $h_{s}$ (the height above sea level) is higher than sea level, a negative elevation angle may occur on the link. Figure 3 shows a schematic presentation of the atmospheric refraction for a negative elevation angle. In this case, the calculation is performed using a segmentation method. First, the spherical angle, $\phi_{1}$, is calculated from the observation point (point $B$ ) to point $A$, in which the ray is parallel to the tangent of the surface of Earth. The spherical angle, $\phi_{2}$, from point A to the satellite (point C) is then calculated. The following relationship can then be obtained:

$$
\phi=\phi_{1}+\phi_{2}
$$

Then, the geometric elevation angle, $\theta_{0}$, can be calculated according to (3):

$$
\theta_{0}=\arctan \left(\frac{r_{3} \cos \phi-r_{1}}{r_{3} \sin \phi}\right)
$$

Based upon Snell's law, described in (1), the following can then be determined:

$$
r_{1} n\left(r_{1}\right) \cos \theta=r_{2} n\left(r_{2}\right) \cos 0^{\circ}=r_{2} n\left(r_{2}\right) .
$$

Then, the apparent elevation angle, $\theta$, can be derived from (12). Finally, $\varepsilon$ can be obtained using (4).

\section{New Formulae for Calculating the Refraction Correction for the Elevation Angle}

Based upon the above methods, the refraction correction for the elevation angle was calculated herein. According to P.834-9 and F.1333-1, the maximum altitude of the station on Earth's surface was set to $3 \mathrm{~km}$, and the calculation interval for altitude was $100 \mathrm{~m}$. For every altitude, when the elevation angle (the apparent elevation angle or the geometric elevation angle) was not less than 0 , the interval of the elevation angle was $0.1^{\circ}$ below $10^{\circ}$, while the interval above $10^{\circ}$ was $1^{\circ}$.

For negative elevation angles, a family of point $\mathrm{A}$ was set to $100 \mathrm{~m}$ intervals, lower than point $\mathrm{B}$ (Figure 3). For example, if the altitude of point $B$ was $300 \mathrm{~m}$, then the heights of the family of point A would be $[200,100,0] \mathrm{m}$.

Based on these calculated data, new formulae for calculating the refraction correction of elevation angle were fitted with the similar forms detailed in P.834-9 and F.1333-1. The height for these new formulae was set to be $100 \mathrm{~km}$ above sea level.

If the apparent elevation angle is known, then 


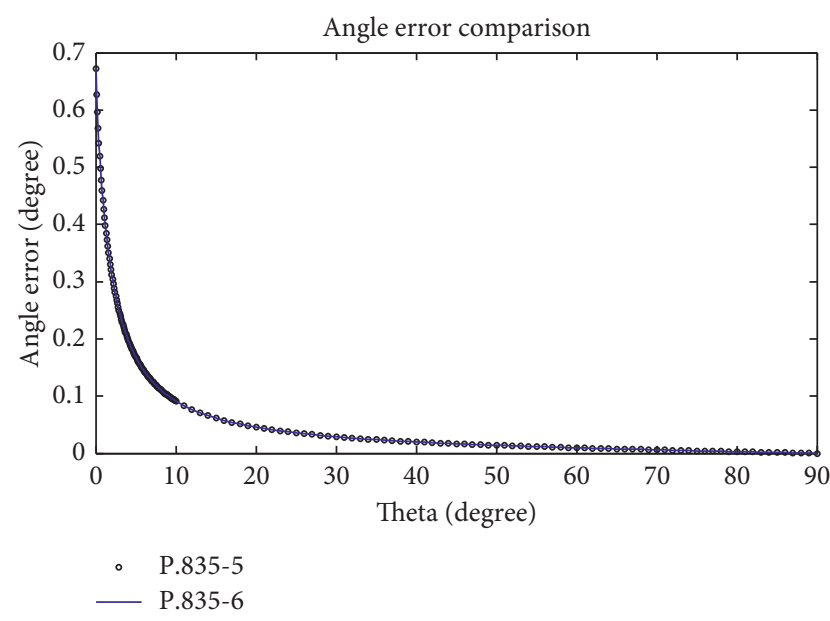

FIGURE 2: Elevation angle errors of two different calculations for the mean annual global reference atmosphere.

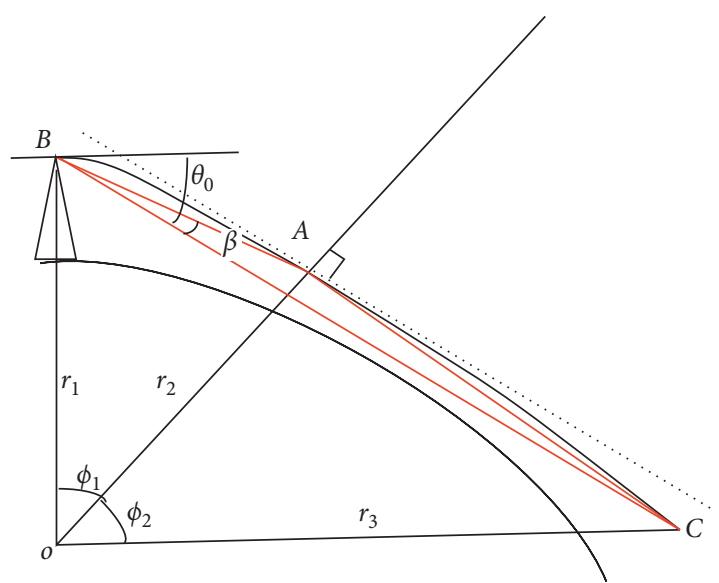

FIgURE 3: Schematic presentation of the atmospheric refraction for the negative elevation angle.

$$
\tau(h, \theta)=\frac{1}{\left[\left(0.00116 h^{2}+0.003247 h+0.01054\right) \theta^{2}+(0.09204 h+0.8445) \theta+(0.3756 h+1.483)\right]} .
$$

If the geometric elevation angle is known, then

$$
\tau\left(h, \theta_{0}\right)=\frac{1}{\left[(0.01721 h+0.02374) \theta_{0}^{2}+\left(0.01601 h^{2}+0.02317 h+0.6126\right) \theta_{0}+(0.2483 h+1.738)\right]},
$$

where $\tau(h, *)$ is the refraction correction for the elevation angle (in degrees).

For satellites at different altitudes, the correction methods are given here based on free-space propagation $[11,12]$. Figure 4 shows a schematic representation of the free-space propagation correction. The refractivity is 0 at points 2 and 4 . Line 03 is perpendicular to the extension of line 42. Based on Snell's law, detailed in (1), the following can be obtained:

$$
r_{3} \sin \gamma=A,
$$

where $\gamma$ is the angle between lines 34 and o4. It should be noted that $\angle 304=\pi / 2-\gamma$; then, the following can be derived:

$$
\phi_{3}=\arccos \frac{A}{r_{3}}-\arccos \frac{A}{r_{2}},
$$

where $\phi_{3}$ is the spherical angle between points 2 and 4 . According to the cosine theorem, the lengths of the line segments of 12,24 , and 14 can be obtained:

$$
\begin{aligned}
& l_{12}=\sqrt{r_{1}^{2}+r_{2}^{2}-2 r_{1} r_{2} \cos \phi}, \\
& l_{24}=\sqrt{r_{2}^{2}+r_{3}^{2}-2 r_{2} r_{3} \cos \phi_{3}},
\end{aligned}
$$

$$
l_{14}=\sqrt{r_{1}^{2}+r_{3}^{2}-2 r_{1} r_{3} \cos \left(\phi+\phi_{3}\right)} .
$$

Meanwhile, the spherical angle $\phi$ cannot be obtained by equation (2) for simplified calculations. In this case, the angle $\angle 2 o 1$ can be obtained by the following equation:

$$
\angle 2 o 1=\frac{\pi}{2}+\theta-\tau(h, \theta) \text {. }
$$

Then, $\angle 12 o$ can be obtained according to the sine theorem:

$$
\angle 12 o=\arcsin \left(\frac{r_{1}}{r_{2}} \sin \angle 2 o 1\right) .
$$

At last, the spherical angle $\phi$ can be calculated by the following equation:

$$
\phi=\pi-\angle 12 o-\angle 2 o 1 .
$$

The unit of angle in the above formulae (18)-(20) is radians.

Finally, $\varepsilon$ can be calculated from triangle $\Delta 214$ according to the cosine theorem:where $l_{12}, l_{14}$, and $l_{24}$ are the lengths of lines 12,14 , and 24 , respectively.

$$
\varepsilon=\arccos \frac{l_{12}^{2}+l_{14}^{2}-l_{24}^{2}}{2 l_{12} l_{14}},
$$




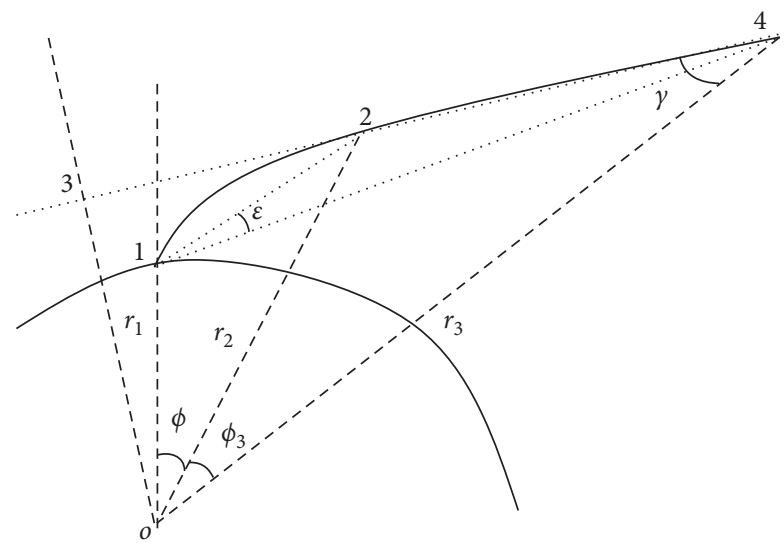

FIgURE 4: A schematic representation of the free-space propagation correction.

For the correction methods when the geometric elevation angle is known (for which the orbit height is set as $100 \mathrm{~km}), l_{12}$ may be obtained by using the sine theorem. The apparent elevation angle can be calculated by (14), after which $A$ in (15) can be obtained. The rest of the calculations are identical to the correction methods that are used when the apparent elevation angle is known.

\section{Comparison of the Different Methods}

Based upon these calculated data, obtained via the raytracing method, the proposed new formulae are compared with the methods detailed in the Recommendations. Table 1 shows the errors for geosynchronous orbit satellites when the apparent elevation angle is known. These errors include the mean and root mean square (RMS) values. In the error calculation process, the results of the ray-tracing method are taken to be the true values, and all the points in Section 3 are used as the total dataset for the error calculation. The new method greatly improves the prediction accuracy.

Figure 5 shows a comparison of the different methods, at sea level and for low elevation angles (below $5^{\circ}$ ), when the apparent elevation angle is known. The new method shows the best agreement with regard to the results of the ray tracing.

Moreover, this paper also presents calculations of the elevation errors for orbital heights of $1,000 \mathrm{~km}$ and $100 \mathrm{~km}$, respectively, and compares them with these models. Tables 2 and 3 show the errors for satellites with orbital heights of $1,000 \mathrm{~km}$ and $100 \mathrm{~km}$, respectively, when the apparent elevation angle is known. The new method exhibits a greatly improved prediction accuracy. It should be noted, however, that the models of the two ITU Recommendations exhibit better results when the orbit is higher compared with themselves.

When the geometric elevation angle is known, the errors cannot be calculated directly by (14) and the
TABLE 1: Errors for geosynchronous orbit satellites when the apparent elevation angle is known (in degrees).

\begin{tabular}{lcc}
\hline Method & Mean & RMS \\
\hline New & 0.0006 & 0.0027 \\
P.834-9 & -0.0038 & 0.0134 \\
F.1333-1 & -0.0025 & 0.0098 \\
\hline
\end{tabular}

corresponding correction methods because the geometric elevation angle in (14) applies only to cases where the orbit height is $100 \mathrm{~km}$. For other orbit heights, the geometric elevation angle applicable to (14) can be determined using the binary search method (a half-interval search algorithm) given a known geometric elevation angle at the present orbit height. Table 4 shows the errors for geosynchronous orbit satellites when the geometric elevation angle is known, including the mean and RMS values. The new method exhibits a greatly improved prediction accuracy.

It should be noted that the methods in this study are developed by the new mean annual global reference atmosphere. The applicability of the method in this paper is analyzed using the other two reference atmospheres provided by ITU-R [4]: low-latitude annual reference atmosphere and winter high-latitude reference atmosphere, which represent two extreme cases. Tables 5 and 6 show the errors for satellites with orbital heights of $36,000 \mathrm{~km}$ for the two kinds of the atmosphere, respectively, when the apparent elevation angle is known. It can be seen from the tables that all three methods have larger errors for the low-latitude annual reference atmosphere and smaller errors for the winter high-latitude reference atmosphere; meanwhile, these errors are all larger than those in Table 1 for the mean annual global reference atmosphere. On the contrary, the root mean square errors of the three methods are very close. 


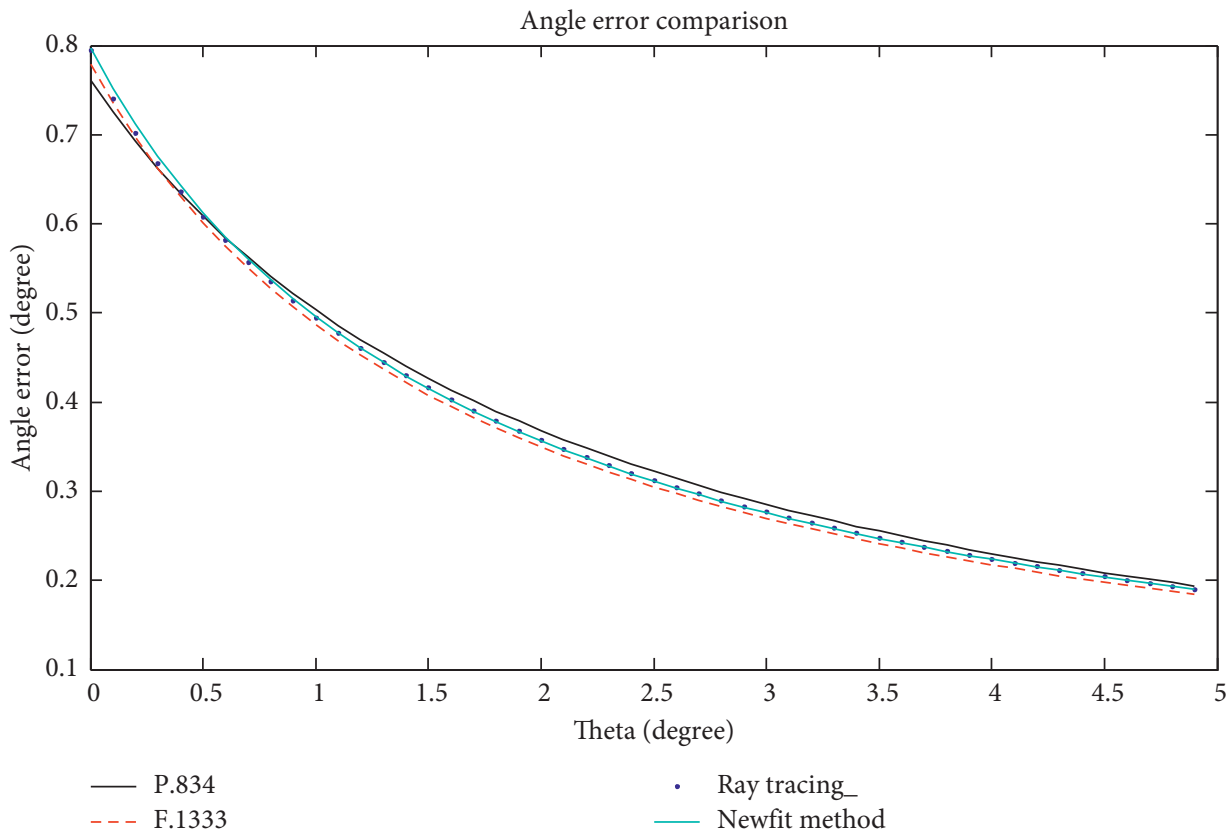

Figure 5: Comparison of the different methods at sea level and below $5^{\circ}$.

TABLE 2: Errors for an orbit of $1,000 \mathrm{~km}$ when the apparent elevation angle is known (in degrees).

\begin{tabular}{lcc}
\hline Method & Mean & RMS \\
\hline New & 0.0018 & 0.0062 \\
P.834-9 & 0.0048 & 0.0189 \\
F.1333-1 & 0.0061 & 0.0138 \\
\hline
\end{tabular}

TABLE 3: Errors for an orbit of $100 \mathrm{~km}$ when the apparent elevation angle is known (in degrees).

\begin{tabular}{lcc}
\hline Method & Mean & RMS \\
\hline New & 0.0001 & 0.0023 \\
P.834-9 & 0.0230 & 0.0498 \\
F.1333-1 & 0.0243 & 0.0462 \\
\hline
\end{tabular}

TABLE 4: Errors for geosynchronous orbit satellites when the geometric elevation angle is known (in degrees).

\begin{tabular}{lcc}
\hline Method & Mean & RMS \\
\hline New & 0.0003 & 0.0043 \\
P.834-9 & -0.0023 & 0.0113 \\
F.1333-1 & -0.0025 & 0.0097 \\
\hline
\end{tabular}

TABLE 5: Errors for an orbit of $36,000 \mathrm{~km}$ for the low-latitude annual reference atmosphere when the apparent elevation angle is known (in degrees).

\begin{tabular}{lcc}
\hline Method & Mean & RMS \\
\hline New & -0.0506 & 0.1291 \\
P.834-9 & -0.0615 & 0.1373 \\
F.1333-1 & -0.0607 & 0.1417 \\
\hline
\end{tabular}

TABle 6: Errors for an orbit of $36,000 \mathrm{~km}$ for the winter highlatitude reference atmosphere when the apparent elevation angle is known (in degrees).

\begin{tabular}{lcc}
\hline Method & Mean & RMS \\
\hline New & 0.0035 & 0.0238 \\
P.834-9 & -0.0006 & 0.0140 \\
F.1333-1 & 0.0009 & 0.0173 \\
\hline
\end{tabular}

\section{Conclusion}

In some studies examining frequency sharing between fixed service and space radiocommunication services, the apparent elevation angle of a space station must be estimated, while accounting for atmospheric refraction. Recommendations ITU-R P.834-9 and F.1333-1 detail similar methods regarding calculating the refraction correction for the elevation angle of the mean annual global reference atmosphere. Herein, both methods are approximated using the bending angle from the ground to the infinity height. This approach is most suitable for geosynchronous orbit satellites. On the contrary, the mean annual global reference atmosphere given in Recommendation ITU-R P.835 was modified in 2017 (P.835-6) by explicitly defining the pressure at the transitions between various layers.

In this paper, new methods for calculating the refraction correction for the elevation angle are proposed regarding the mean annual global reference atmosphere given in Recommendation ITU-R P.835-6. These methods were obtained by fitting the results of the ray-tracing method, which included two situations: either the apparent elevation angle is known or the geometric elevation angle is known. The height of the new formulae is $100 \mathrm{~km}$ above sea level. For higher 
altitudes, here, correction methods are presented based on free-space propagation. The proposed methods can be used to calculate the refraction correction for the elevation of the mean annual global reference atmosphere for satellites at different orbital heights. The analysis and comparisons presented in Section 4 show that the new method effectively improves the prediction accuracy of the refraction correction for the elevation angle of the new mean annual global reference atmosphere at various orbit heights.

It should be noted that the methods used in this study are mainly applicable to the new mean annual global reference atmosphere or atmospheres with similar conditions. The errors of the method in this study are increased when it is applied to two other ITU-R reference atmospheres. For the calculation of the refraction correction of elevation angles under actual atmospheric profiles, further studies are needed. For a spherical stratified atmosphere in a typical climatic zone, calculation formulae similar to our study can be established. For more complicated cases where the refractivity of the atmosphere is unevenly distributed in the horizontal direction, it should be calculated using the raytracing method.

\section{Data Availability}

There are no experimental data in the manuscript. All calculations in the manuscript rely on the atmospheric profiles given in the standard atmospheric Recommendations (Recommendations ITU-R P.835-5 and P.835-6) and the ray-tracing algorithms given in the manuscript. Recommendations ITU-R P.835-5 and P.835-6 can be found on the website https://www.itu.int/rec/R-REC-P.835/en and can be downloaded for free.

\section{Conflicts of Interest}

The authors declare that they have no conflicts of interest.

\section{Acknowledgments}

The authors gratefully acknowledge the support from the National Natural Science Foundation of China (NSFC) under Grant nos. 61971385 and 61901424. The authors would also like to thank Editage (http://www.editage.com) for English language editing.

\section{References}

[1] Y. Xie, J. Lavergnat, J. P. S. Mon et al., Microwave and Millimetre Wave Propagation, International Academic Publishers, Beijing, China, 1995.

[2] Recommendation ITU-R F.1333-1, Estimation of the Actual Elevation Angle from a Station in the Fixed Service Towards a Space Station Taking into Account Atmospheric Refraction, International Telecommunication Union, Geneva, Switzerland, 1999.

[3] Recommendation ITU-R P.834-9, Effects of Tropospheric Refraction on Radiowave Propagation, International Telecommunication Union, Geneva, Switzerland, 2017.
[4] Recommendation ITU-R P.835-6, Reference Standard Atmospheres, International Telecommunication Union, Geneva, Switzerland, 2017.

[5] M. Born and E. Wolf, Principles of Optics, Pergamon Press, New York, NY, USA, 1964.

[6] A. R. Lowry, C. Rocken, S. V. Sokolovskiy et al., "Vertical profiling of atmospheric refractivity from ground-based GPS," Radio Science, vol. 37, no. 3, 2002.

[7] Recommendation ITU-R P.834-2, Effects of Tropospheric Refraction on Radiowave Propagation, International Telecommunication Union, Geneva, Switzerland, 1997.

[8] Recommendation ITU-R F.1333-0, Estimation of the Actual Elevation Angle from a Station in the Fixed Service Towards a Space Station Taking into Account Atmospheric Refraction, International Telecommunication Union, Geneva, Switzerland, 1997.

[9] Recommendation ITU-R P.453-13, The Radio Refractive Index: Its Formula and Refractivity Data, International Telecommunication Union, Geneva, Switzerland, 2017.

[10] Recommendation ITU-R P.835-5, Reference Standard Atmospheres, International Telecommunication Union, Geneva, Switzerland, 2012.

[11] Y. M. Liu, X. M. Chen, Z. W. Zhao et al., "High-precision fast ray-tracing algorithm used in optical wave refraction error correction system," Chinese Journal of Radio Science, vol. 30, no. 4, pp. 749-753, 2015.

[12] Z. M. Zhu, H. N. Li, Y. X. Huang et al., "An exact model and on-line algorithm for radio refraction error correction," System Engineering and Electronics, vol. 25, no. 3, pp. 287-289, 2003. 